\title{
Probing annihilations and decays of low-mass galactic dark matter by track and cascade events in IceCube DeepCore
}

\author{
Fei-Fan Lee ${ }^{* \dagger}$ \\ Institute of Physics, National Chiao-Tung University, Hsinchu 30010, Taiwan \\ E-mail: fflee@mail.nctu.edu.tw

\section{Guey-Lin Lin} \\ Institute of Physics, National Chiao-Tung University, Hsinchu 30010, Taiwan \\ E-mail: glin@mail.nctu.edu.tw
}

\begin{abstract}
The deployment of DeepCore array significantly lowers IceCube's energy threshold to about 10 $\mathrm{GeV}$ and enhances the sensitivity of detecting neutrinos from annihilations and decays of light dark matter. To match this experimental development, we provide a complete analysis of track and cascade event rates in DeepCore array due to neutrino flux produced by annihilations and decays of galactic dark matter. We also calculate the background event rates due to atmospheric neutrino flux for evaluating the sensitivity of DeepCore array to galactic dark matter signatures. Unlike previous approaches, which set the energy threshold for track and cascade events at around $50 \mathrm{GeV}$ (this choice makes the estimation of atmospheric background event rate much simpler, i.e., the oscillation effect in atmospheric neutrino flux can be neglected), we have set the energy threshold at $10 \mathrm{GeV}$ to take the full advantage of DeepCore array. We compare our calculated sensitivity with those obtained by setting a $50 \mathrm{GeV}$ threshold energy. We conclude that our choice of threshold energy significantly improves the sensitivity to dark matter signature for WIMP mass below $100 \mathrm{GeV}$.
\end{abstract}

The 2011 Europhysics Conference on High Energy Physics, EPS-HEP 2011,

July 21-27, 2011

Grenoble, Rhône-Alpes, France

\footnotetext{
${ }^{*}$ Speaker.

${ }^{\dagger}$ This work is supported by the National Science Council of Taiwan.
} 


\section{Introduction}

The installation of DeepCore array significantly improves the rejection of downward going atmospheric muons in IceCube and lowers the threshold energy for detecting muon track or cascade events to about $5 \mathrm{GeV}$. These improvements enhance the sensitivity as well as enlarge the energy window for observing neutrinos from dark matter (DM) annihilations or decays in the galactic halo. To match this experimental development, we first calculate the neutrino fluxes from WIMP annihilations and decays in the galactic halo. We then calculate the atmospheric neutrino fluxes from all zenith angles with $E_{v} \geq 10 \mathrm{GeV}$. The oscillations between $v_{\mu}$ and $v_{\tau}$ are taken into account. We next calculate both track and cascade event rates for evaluating the sensitivity of DeepCore array to neutrino flux arising from WIMP annihilations or decays in the galactic halo. Finally, We compare our results with those obtained by setting $E_{\mu}^{\text {th }}=50 \mathrm{GeV}$.

\section{Analysis and Results}

The differential neutrino flux from the galactic dark matter halo for neutrino flavor $i$ can be written as [1]

$$
\frac{\mathrm{d} \Phi_{v_{i}}}{\mathrm{~d} E_{v_{i}}}=\frac{\Delta \Omega}{4 \pi} \frac{\langle\sigma v\rangle}{2 m_{\chi}^{2}}\left(\sum_{F} B_{F} \frac{\mathrm{d} N_{v_{i}}^{F}}{\mathrm{~d} E}\right) R_{\odot} \rho_{\odot}^{2} \times J_{2}(\Delta \Omega)
$$

for the case of annihilating DM, and

$$
\frac{\mathrm{d} \Phi_{v_{i}}}{\mathrm{~d} E_{v_{i}}}=\frac{\Delta \Omega}{4 \pi} \frac{1}{m_{\chi} \tau_{\chi}}\left(\sum_{F} B_{F} \frac{\mathrm{d} N_{v_{i}}^{F}}{\mathrm{~d} E}\right) R_{\odot} \rho_{\odot} \times J_{1}(\Delta \Omega)
$$

for the case of decaying DM, where $\langle\sigma v\rangle$ is the thermally averaged annihilation cross section, $\tau_{\chi}$ is the DM decay time, and $J_{n}(\Delta \Omega)$ is the DM distribution integrated over the line-of-sight (1.o.s) and averaged over a solid angle $\Delta \Omega=2 \pi\left(1-\cos \psi_{\max }\right)$. Here we use Navarro-Frenk-White (NFW) DM density profile [2] to calculate $J_{n}(\Delta \Omega)$. After that, we employ neutrino flavor oscillation probabilities in the tribimaximal limit of neutrino mixing angles to get the neutrino fluxes on Earth from those at the source.

Knowing atmospheric neutrino background is important for evaluating the sensitivity of DeepCore array to neutrino flux from DM annihilations or decays. We follow the approaches in Ref. [3] to compute the intrinsic atmospheric neutrino fluxes. We find that the angle-averaged atmospheric muon neutrino flux obtained by our calculation agrees well with AMANDA-II results [4]. Subsequently, we can calculate the atmospheric neutrino fluxes taking into account neutrino oscillations.

In IceCube DeepCore, the track event rate for contained muons is given by

$$
\Gamma_{\mu}=\int_{E_{\mu}^{\mathrm{th}}}^{E_{\max }} \mathrm{d} E_{\mu} \int_{E_{\mu}}^{E_{\max }} \mathrm{d} E_{v_{\mu}} N_{A} \rho_{\text {ice }} V_{\mathrm{tr}} \times \frac{d \Phi_{v_{\mu}}}{d E_{v_{\mu}}} \cdot \frac{d \sigma_{v N}^{\mathrm{CC}}\left(E_{v_{\mu}}, E_{\mu}\right)}{d E_{\mu}}+(v \rightarrow \bar{v}),
$$

while the cascade event rate is given by

$$
\Gamma_{\text {casc }}=\int_{E_{s h}^{\text {th }}}^{E_{\max }} \mathrm{d} E_{s h} \int_{E_{s h}}^{E_{\max }} \mathrm{d} E_{v} N_{A} \rho_{\text {ice }} V_{\text {casc }} \times \frac{d \Phi_{v}}{d E_{v}} \cdot \frac{d \sigma_{v N}\left(E_{v}, E_{s h}\right)}{d E_{s h}}+(v \rightarrow \bar{v}),
$$




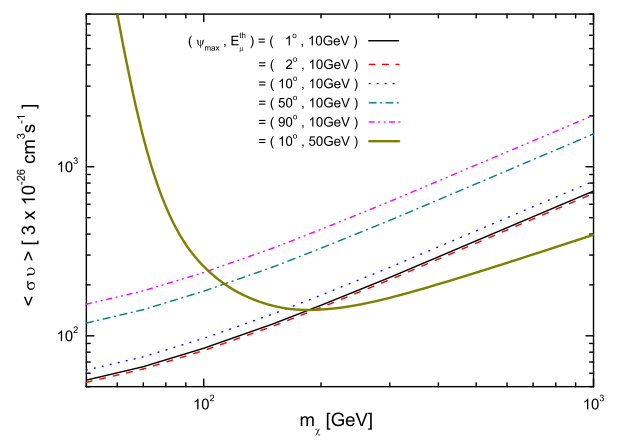

Figure 1: The required DM annihilation cross section $\left(\chi \chi \rightarrow \mu^{+} \mu^{-}\right)$as a function of $m_{\chi}$ such that the neutrino signature from DM annihilations can be detected at the $2 \sigma$ significance in five years for track events. Results corresponding to different $\psi_{\max }$ are presented. For comparison, we also show the result with $E_{\mu}^{\text {th }}=50 \mathrm{GeV}$ and $\psi_{\max }=10^{\circ}[1]$.

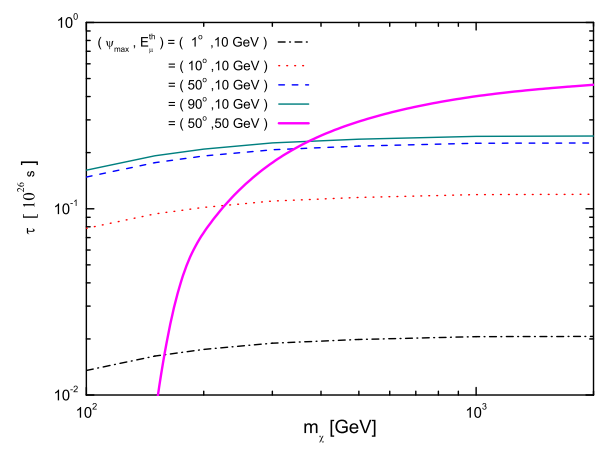

Figure 2: The required DM decay time $\left(\chi \rightarrow \mu^{+} \mu^{-}\right)$as a function of $m_{\chi}$ such that the neutrino signature from DM decays can be detected at the $2 \sigma$ significance in five years for track events. For comparison, we also show the result with $E_{\mu}^{\mathrm{th}}=50 \mathrm{GeV}$ and $\psi_{\max }=50^{\circ}[1]$.

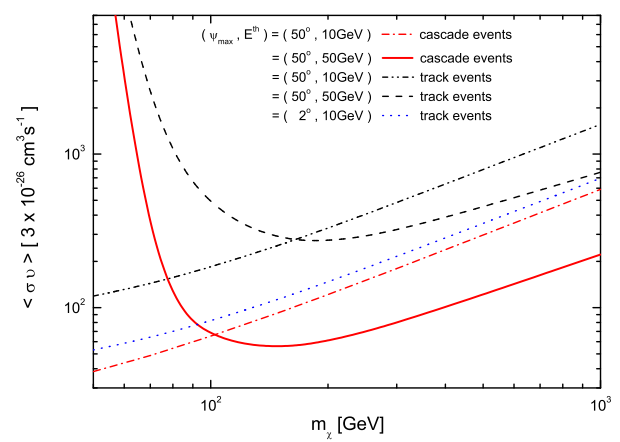

Figure 3: The required DM annihilation cross section $\left(\chi \chi \rightarrow \mu^{+} \mu^{-}\right)$as a function of $m_{\chi}$ such that the neutrino signature from DM annihilations can be detected at the $2 \sigma$ significance in five years for track and cascade events. 
where $V_{\mathrm{tr}} \approx 0.04 \mathrm{~km}^{3}$ is the effective volume of IceCube DeepCore array for muon track events and $V_{\text {casc }} \approx 0.02 \mathrm{~km}^{3}$ is that for cascade events.

Here we take the leptophilic model with DM predominantly annihilates into $\mu^{+} \mu^{-}$for illustration. By computing the track event rates, we present in Fig. 1 the required DM annihilation cross section as a function of $m_{\chi}$ with a low threshold energy $E_{\mu}^{\text {th }}=10 \mathrm{GeV}$ for different cone half-angle $\psi_{\max }$ such that the neutrino signature from DM annihilations can be detected at the $2 \sigma$ significance in five years. Non-detection of such a signature would then exclude the parameter region above the curve. The result of DM decays is shown in Fig. 2. Non-detection of DM decay signature would then exclude the parameter region below the curve. For details, see Ref. [4].

In Fig. 3, we compare the DM annihilation constraints given by cascade events and track events. We note that $v_{\mu}$ is the dominant flavor of atmospheric neutrinos above few tens of $\mathrm{GeV}$ and the neutrino-nucleon cross section are almost the same for all flavors. Therefore, comparing with the result of track events, the signal to background ratio is enhanced in cascade events because $v_{\mu}$ only produces the cascade events through the neutral-current interaction, which is lower in cross section than that of charged-current interaction. This is why cascade events in general provide stronger constraints on DM annihilation cross section than that given by track events. However, we can see in Fig. 3 that the curve of track events for $\psi_{\max }=2^{\circ}$ is close to that of cascade events. In other words, for DM annihilations, the sensitivity of track events for sufficiently small cone half-angle could be comparable to the sensitivity of cascade events.

\section{Summary}

We employ NFW DM profile and leptophilic DM model to calculate the track and cascade event rates in IceCube DeepCore caused by neutrino fluxes from DM annihilations and decays in the galactic halo. In addition, we also take into account neutrino oscillations and calculate the event rates due to atmospheric neutrino flux.

For track events, we compare our calculated sensitivity based upon $E_{\mu}^{\text {th }}=10 \mathrm{GeV}$ with those obtained by taking $E_{\mu}^{\text {th }}=50 \mathrm{GeV}$ and conclude that our choice of $E_{\mu}^{\text {th }}=10 \mathrm{GeV}$ significantly improves the sensitivity to DM signature for $m_{\chi}<100 \mathrm{GeV}$ in the annihilation channel and $m_{\chi}<$ $300 \mathrm{GeV}$ in the decay channel. On the other hand, cascade events provide stronger constraints on DM annihilation cross section and DM decay time than the corresponding constraints provided by track events with the same threshold energy. The sensitivity of track events to DM annihilation cross section could be comparable to that of cascade events provided the cone half-angle taken for track events is sufficiently small.

\section{References}

[1] A. E. Erkoca, M. H. Reno, and I. Sarcevic, Phys. Rev. D 82, 113006 (2010).

[2] J. F. Navarro, C. S. Frenk and S. D. M. White, Astrophys. J. 462, 563 (1996).

[3] T. K. Gaisser, Astropart. Phys. 16, 285 (2002); F. F. Lee and G. L. Lin, Astropart. Phys. 25, 64 (2006).

[4] F. F. Lee and G. L. Lin, arXiv:hep-ph/1105.5719. 